\title{
Skin-Sparing Mastectomy
}

National Cancer Institute

\section{Source}

National Cancer Institute. Skin-Sparing Mastectomy. NCI Thesaurus. Code C107211.

Removal of the breast tissue, nipple, areola, and original biopsy scar, but not the breast skin. 